\title{
Bit Error Rate Performance of Spread Spectrum Communication Systems for AWGN Channels
}

\author{
Revathy R., P. R. Saseendran Pillai
}

\begin{abstract}
The digital communication technologies have gained immense significance as it provides secure and error free services. One of the major advantages of digital communication is that they are much resistant to transmitted as well as interpreted errors. For ensuring the security of data, the most suitable method is to use spread spectrum technique. The spread spectrum technique has gained immense popularity for use in various systems as the spreading of the spectral bandwidth offer many advantages, including the establishment of secure communications, increasing resistance to interference, noise rejection, and so on. The signals which are modulated by using these techniques cannot be jammed and are very hard to interfere. This paper presents the results of investigation of BPSK based direct sequence spread spectrum systems for Additive White Gaussian Noise (AWGN) and undersea channels. The bit error rate performance of BPSK based direct sequence spread spectrum systems has been simulated for the AWGN channel and the results have been plotted.
\end{abstract}

Keywords: Spread spectrum, AWGN, BPSK, Bellhop

\section{INTRODUCTION}

For an error free and effective communication to occur, the receiver should be able to receive the exact data sent by the transmitter, even when the signal is buried in high noise fields. The interference of the noise signal in the communication channel which can be either intentional or unintentional can reduce its efficiency. In such situations spread spectrum communication [3, 4] can be used for reliable and secure data transmissions, even in a noisy environment. Spread spectrum is a wide band technique in which the signal generated with a particular bandwidth is purposely spread in the frequency domain using a spreading code which is known only to the transmitter and receiver, resulting in a signal with a wider bandwidth, which appears to be noise like for unauthorized users. By sending the signal at low power it can be hidden in the channel as noise. This is achieved by spreading the signal energy over the widest bandwidth assessable and by using the less power levels. As a result the probability of detecting the signal will be low for

Revised Manuscript Received on February 05, 2020.

* Correspondence Author

Revathy R.*, Department of Electronics, Cochin University of Science and Technology, Kochi-682022, India. E-mail: revathyradha2010@gmail.com

P. R. Saseendran Pillai, Department of Electronics, Cochin University of Science and Technology, Kochi-682022, India. E-mail: prspillai@cusat.ac.in

(C) The Authors. Published by Blue Eyes Intelligence Engineering and Sciences Publication (BEIESP). This is an open access article under the CC BY-NC-ND license (http://creativecommons.org/licenses/by-nc-nd/4.0/) unintended receiver other than the intended receiver. The main advantages of using spread spectrum communications are interference suppression, ability to provide multiple accesses, energy density reduction and fine time resolution. The overall signal interference can be reduced by the efficient use of the direct sequence spread spectrum modulation technique. By way of spreading the signal using the spreading code makes it look like noisy waveforms, thus this technique offers greater resistance to unintentional and intentional interferences.

\section{SYSTEM MODEL}

Performance of spread spectrum communication systems mainly relies on the factor that, the bandwidth required for the signal transmission should be much larger than the least bandwidth required for information transmission and also should be spread by a spreading code which is independent of the data. This code has pseudo random properties and is known to the receiver prior to transmission. Demodulation is then performed by correlating the received code with an exact copy of the spreading code in the receiver; and hence the signal is de-spread at the receiver side.

The overall signal interference can be reduced by direct sequence spread spectrum signals. The block diagram of a BPSK based direct sequence spread spectrum system for an AWGN channel is given in Fig 1.

The input data signal $\mathrm{m}(\mathrm{t})$ is spread using the spreading code designed $\mathrm{c}(\mathrm{t})$, resulting spread signal $\mathrm{s}(\mathrm{t})$ is later modulated by the binary phase shift keying (BPSK) modulator. The output of the BPSK modulator $\mathrm{x}(\mathrm{t})$ is transmitted over the AWGN channel and the received signal is de- spread using the same spreading code.

The spreading code should be properly selected and designed.

Also by efficient design of codes multiple systems can co-exist without any interference. 


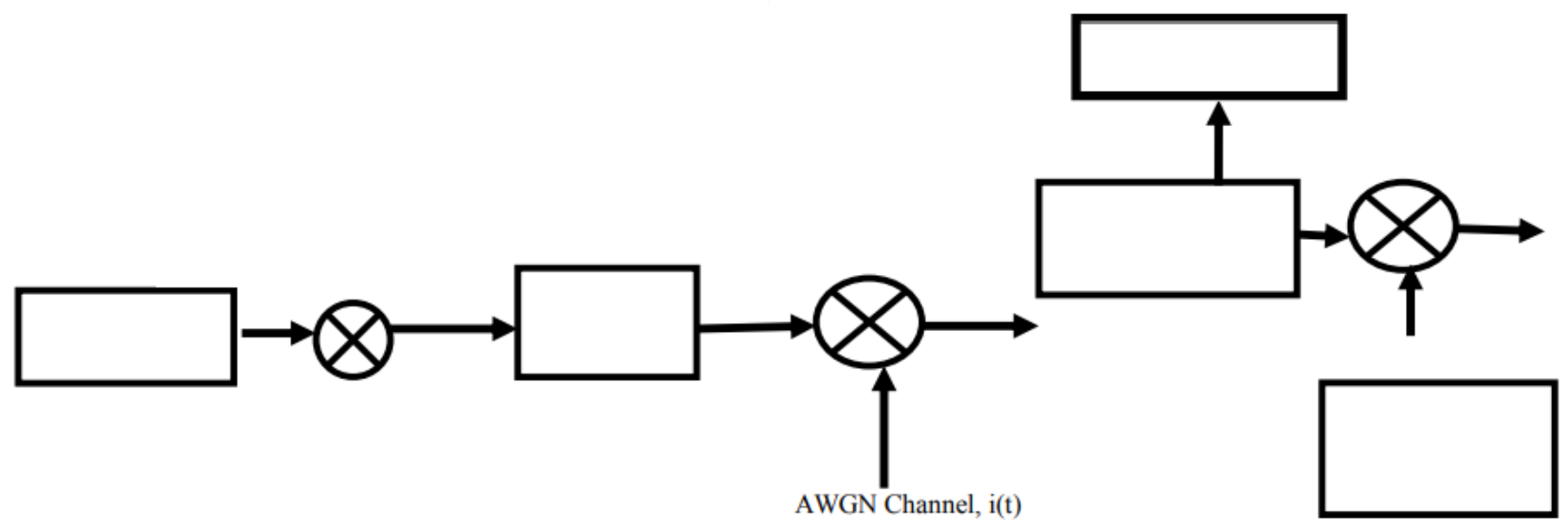

Fig 1:- Block diagram of BPSK based Spread Spectrum System

\section{RESULTS AND DISCUSSIONS}

\section{A. Simulation of AWGN Channel}

Additive white Gaussian noise (AWGN) is simple noise model used to mimic the effect of random processes. It is mentioned as additive because they can be added to any noise that might be intrinsic to the information system. Further they are not much affected by fading, frequency selectivity, interference, nonlinearity or dispersion and so on. The parameters used for simulation are furnished in Table 1.

Table 1: simulation parameters used

\begin{tabular}{|l|l|l|}
\hline Parameters & $\begin{array}{l}\text { Without } \\
\text { DSSS }\end{array}$ & With DSSS \\
\hline $\begin{array}{l}\text { No. of transmitted } \\
\text { bits }\end{array}$ & $10^{6}$ & $10^{8}$ \\
\hline $\begin{array}{l}\text { Modulation } \\
\text { scheme used }\end{array}$ & BPSK & BPSK \\
\hline $\begin{array}{l}\text { Frequency of } \\
\text { carrier }\end{array}$ & $10 \mathrm{kHz}$ & $10 \mathrm{kHz}$ \\
\hline $\begin{array}{l}\text { Signal frequency } \\
\text { band: BW }\end{array}$ & $10 \mathrm{kHz}$ & $10 \mathrm{kHz}$ \\
\hline
\end{tabular}

The BER implementation of the direct sequence spread spectrum communication system for an AWGN channel is shown in Fig 2.

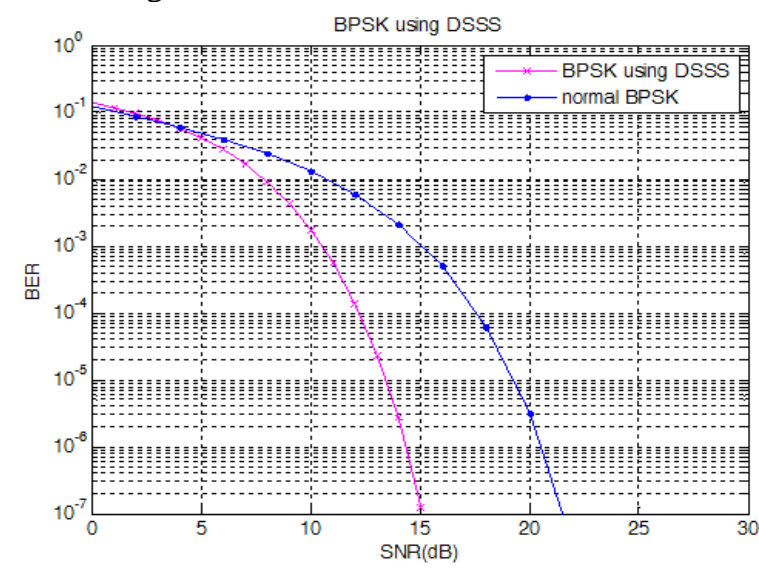

Fig 2:- BER plot of BPSK based DSSS and normal BPSK in Table 2.

Table 2: BER values obtained for normal BPSK and DSSS based BPSK

\begin{tabular}{|l|l|l|}
\hline SNR $(\mathrm{dB})$ & $\begin{array}{l}\text { BER values for } \\
\text { Normal BPSK }\end{array}$ & $\begin{array}{l}\text { BER values for } \\
\text { BPSK based } \\
\text { DSSS }\end{array}$ \\
\hline 0 & 0.1256 & 0.1392 \\
\hline 3 & 0.0858 & 0.7742 \\
\hline 5 & 0.0606 & 0.4189 \\
\hline 8 & 0.0328 & 0.0092 \\
\hline 13 & 0.0243 & $0.4232 \times 10^{-5}$ \\
\hline 14 & 0.0133 & $0.2763 \times 10^{-6}$ \\
\hline 15 & 0.0051 & $0.8421 \times 10^{-8}$ \\
\hline
\end{tabular}

\section{B. Simulation of Undersea Channel Effects}

Acoustic propagation is effective at low frequencies, and the available bandwidth is extremely limited [6]. The major factors that affect the undersea acoustic propagation are signal frequency which increases the attenuation, time-varying multipath propagation, and low speed of sound. The available bandwidth, range and signal-to-noise ratio are determined by the transmission loss and noise.

$$
\text { Transmission loss }=10 \log r
$$

where $r$ is the range from the transmitter. In undersea communication, the major cause resulting in multipath effects are due to the sound reflection at the surface, bottom, and also any objects, and the refraction of sound in the water. Time variability of the channel is the result of implicit changes in the propagation medium such as the changes in temperature and of the motion of the transmitter and receiver. Major variations caused are by surface waves, which results in the displacement of the reflection point, and further leads to scattering and Doppler spreading of the signal due to the change in path length. The additional features that contribute to the changes in the channel impulse response are the motion of the transmitter and the receiver which causes frequency shifting along with additional frequency shifting, due to Doppler Spread. 
In typical undersea acoustic links, the normalized Doppler spread can converge on a value of about $10^{-2}$. Channel coding is important to protect the transmitted signal against noise and all other impairments in underwater channels. A study has been made to generate the effects of undersea channel based on BPSK based direct sequence spread spectrum signals, by the Bellhop tool box.

\section{Bellhop Model}

The Bellhop [1]-[2] model, which is an acoustic tool box, is used for simulation of undersea acoustic communication as the sea trials are very expensive and time consuming. Transmission loss, channel impulse response, sound speed profile, etc. can be generated by this tool box. An exact description of the features of the target environment is highly recommended for better performance. This includes the sound speed profile, depth of the ocean, and the data regarding the bottom outline and roughness, surface etc., and can be gathered from in situ measurements under known environmental conditions. Open ocean measurements will ensure that the model produces more realistic results. The operational frequency, speed-depth pairs, numbers of sources, receivers their depths etc... should be specified in the environmental file mentioned. The resulting response is convolved with the signal to be transmitted for modeling the multipath propagation effects in the oceanic environment.

\section{Sound Speed Profile}

The sound speed profile, known as SSP in sea water determines the behavior of sound propagation in the sea and varies with location, depth, temperature, etc... The sound speed profile depicts a curve of the sound speed with depth. Propagation of sound is also affected by the variations in the speed of sound in the oceanic environment and is under construction. The speed of the sound in ocean is given by Leroy's formula,

$\mathrm{c}=1492.9+3(\mathrm{~T}-10)-6 \times 10^{-3}(\mathrm{~T}-10)^{2}-4 \times 10^{-2}(\mathrm{~T}-18)^{2}+$ $1.2(\mathrm{~S}-35)-10^{-2}(\mathrm{~T}-18)+(\mathrm{S}-35)+\mathrm{z} / 16$.

where $\mathrm{c}$ is the sound speed in meters per second, $\mathrm{T}$ is the temperature in degree centigrade, $\mathrm{S}$ is the salinity in parts per thousand and $\mathrm{z}$ is the depth in meters. The sound speed profile and the normalized channel impulse response plotted using the Bellhop model is shown in Fig 3. The Sound Speed Profile for an oceanic environment with known CTD (conductivity, temperature and depth) data are computed using the equation (2).

The channel impulse response (CIR) shown in Fig 4 has been generated with the help of Bellhop based on a modeled undersea scenario. The given sound speed profile performs the multipath propagation modeling by tracing number of rays through the undersea channel. As the speed of the sound varies with depth the rays no longer travel on straight lines. Bellhop toolbox is used to generate the impulse response of the channel, fixing source depth at $50 \mathrm{~m}$, receiver depth at $100 \mathrm{~m}$, and receiver range and ocean depth at $1 \mathrm{~km}$.

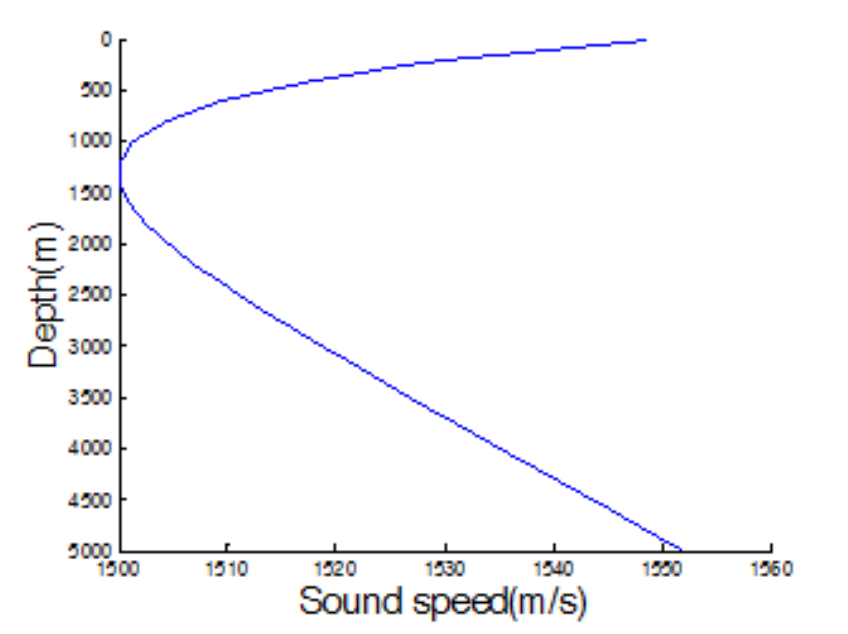

Fig 3:- Sound speed profile of underwater acoustic channel

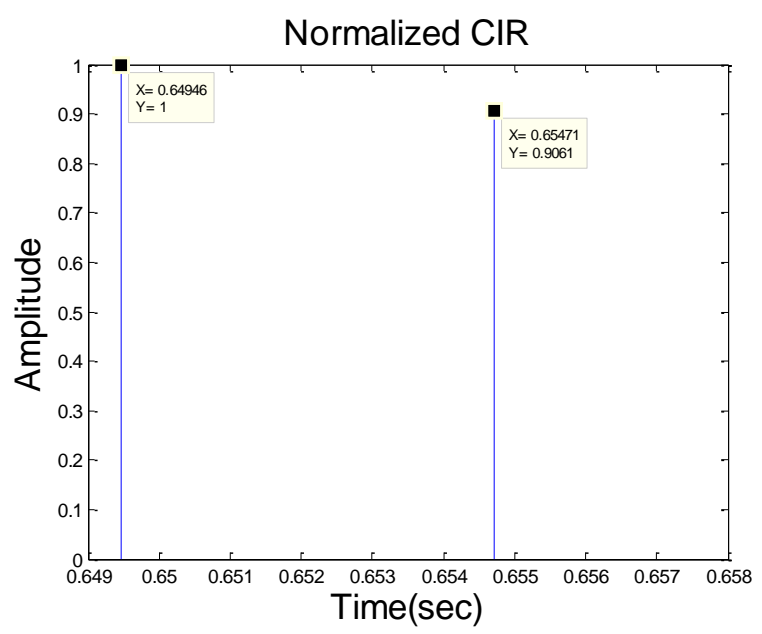

Fig 4:- Channel impulse response for underwater acoustic channel

\section{CONCLUSION}

BPSK based direct sequence spread spectrum communication system and normal BPSK has been simulated under the AWGN channel environment and the bit error rates have been obtained and the results are plotted. The results clearly indicates that the spread spectrum based BPSK has much improved performance than normal BPSK. For an SNR of $15 \mathrm{~dB}$ bit error rate for BPSK based spread spectrum systems is found to be $0.8421 \times 10^{-8}$ whereas for normal BPSK the value obtained is 0.0051 .

The undersea channel effects are being simulated using the Bellhop model to investigate the acceptability of spread spectrum based communication systems for the realization of undersea acoustic networks, capable of handling different types of interfering signals, multipath effects, time varying channel effects, bandwidth constraints and strong signal attenuations that can override the effects caused by intentional jammers. 
Bit Error Rate Performance of Spread Spectrum Communication Systems for AWGN Channels

\section{ACKNOWLEDGMENT}

The authors gratefully acknowledge the Department of Electronics, Cochin University of Science and Technology, for extending all the facilities for carrying out this work.

\section{REFERENCES}

1. M.C. Domingo, "Overview of channel models for underwater wireless communication networks", Elsevier, Phy. Communication. vol 1., no.3.,pp 163-182. 2008

2. M. B. Porter, “The BELLHOP Manual and Users Guide," Heat, Light and Sound Research, Inc., La Jolla, CA, USA, 2011

3. S. Shinly Swarna Sugi, Vijesh Joe C, "Spread Spectrum Modulation Techniques using MATLAB", International Journal of Innovative Research in Technology., vol. 2, issue 3, pp. 47-52, 2015.

4. Sanjay Deshmukh, Udhav Bhosle, "Performance Evaluation of Spread Spectrum System Using Different Modulation Schemes", International Conference on Computational Modeling and Security (CMS 2016), Procedia Computer Science 85.,pp 176 - 182. 2016

5. J.G. Prokais, "Communication Systems Engineering", Prentice Hall, 1994, chap 1.

6. Milica Stojanovic, James Preisig, "Underwater Acoustic Communication Channels: Propagation Models and Statistical Characterization," IEEE Communications Magazine., pp. 84-89., jan 2009.

\section{AUTHORS PROFILE}

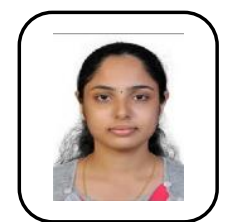

Revathy R., received the BSc. degree in Electronics with Computer Hardware from M. G. University, Kottayam, India in the year 2011 and the MSc. degree in Applied Electronics from the M. G. University, Kottayam, Kochi, India in 2014. She is currently working towards the Ph.D. degree in Underwater Acoustic Communication Networks at Cochin University of science and Technology, Kochi, India, since August 2014. Her research interests include Signal Processing, Undersea Acoustics, Underwater Acoustic Communication, Communication Networks etc.

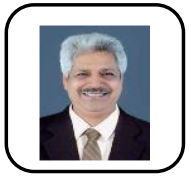

P. R. Saseendran Pillai, started his career as Scientific Officer in the Department of Electrical Communication Engineering, Indian Institute of Science, Bangalore in 1982. In 1983, he joined the Department of Electronics, Cochin University of Science and Technology (CUSAT) as faculty and has been elevated to the post of Professor in Electronics in 1994. He was the Professor and Head of the Department of Electronics, Cochin University of Science and Technology during 1998 to 2001 and 2010 to 2012. Dr.Pillai has secured the Sir C. V. Raman Award for the Best Paper published in the Journal of the Acoustical Society of India in 2002 